\title{
Traduire
}

Revue française de la traduction

$231 \mid 2014$

A table !

\section{La langue des vignes. Vitis vinifera, une liane invasive à la terminologie exubérante}

Dominic Michelin

\section{(2) OpenEdition}

Journals

Édition électronique

URL : http://journals.openedition.org/traduire/657

DOI : 10.4000/traduire.657

ISSN : 2272-9992

Éditeur

Société française des traducteurs

Édition imprimée

Date de publication : 1 décembre 2014

Pagination : 20-39

ISSN : 0395-773X

\section{Référence électronique}

Dominic Michelin, «La langue des vignes. Vitis vinifera, une liane invasive à la terminologie exubérante

», Traduire [En ligne], 231 | 2014, mis en ligne le 01 décembre 2016, consulté le 19 avril 2019. URL :

http://journals.openedition.org/traduire/657 ; DOI : 10.4000/traduire.657 


\section{La langue des vignes \\ Vitis vinifera, une liane invasive à la terminologie exubérante}

\section{Dominic Michelin}

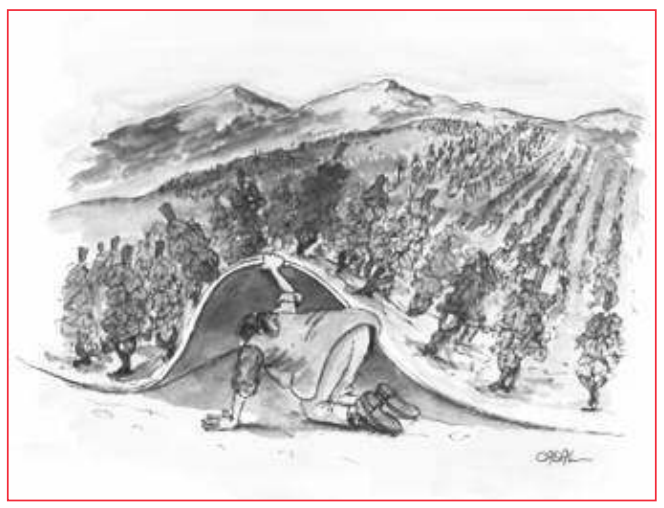

Illustration de Casal parue dans Le Nouvelliste (Suisse) et réalisée pour le site

http://www.sigales.fr/etudes_sols_terroirs/contacts_sigales.html
Seule, dans le règne végétal, la vigne nous rend intelligible ce qu'est la véritable saveur de la terre. Quelle fidélité dans la traduction! Elle ressent, exprime par la grappe les secrets du sol. Colette(1)

Quel meilleur aphorisme pour lancer une réflexion sur la traduction liée au domaine de la culture de la vigne et aux nombreux sous-domaines qui en émanent ? L'univers de la vigne et du vin constitue en soi un véritable vivier terminologique, culturel et sociologique parfois digne d'une jungle. Aussi, comme avant d'introduire toute culture sur un terrain encore sauvage et touffu, il convient de procéder à un défrichage à la fois historique, botanique, sémantique et terminologique.

Avant de devenir traducteur et de me spécialiser dans certains domaines agronomiques dont celui de la vigne et du vin, j'ai occupé différents postes liés au commerce et à la communication dans un cadre international, notamment pour un négociant en vins de Côte-d'Or. Mon travail, outre le suivi des commandes et la prise en charge des clients étrangers dans la triple combinaison linguistique français-anglais-espagnol, consistait à faire visiter les parcelles et la cave ancestrale du domaine et à animer les dégustations, autant dire une mission récurrente d'interprétation qui nécessite de bien connaître non seulement les termes appropriés mais aussi - et surtout - l'» amont " du vin (origine, terrain, culture, histoire) afin de bien pouvoir

(1) Prisons et Paradis, 1932, Paris, J. Ferenczi \& Fils, p. 71. 
parler de l'" aval ", le jeu de mots sur ce dernier terme étant évidemment fortuit, puisque comme chacun sait, lors de la dégustation, le vin est servi, observé, humé, goûté, et bien sûr recraché !

Depuis que je suis traducteur, j'ai eu l'opportunité - et le plaisir - d'utiliser ces connaissances et cette expérience en différentes occasions : traduction d'un questionnaire d'examen en sommellerie, retranscription de scripts d'un film sur les dégustations de porto, communiqués de presse pour une maison de champagne ou encore comme interprète pour une tonnellerie de ma région, mission qui m'a notamment rappelé l'importance extrême du contenant, le fût en l'occurrence, dans la qualité d'un vin prestigieux. L'objet de cet article est donc de donner un aperçu de la typicité de ce domaine et du vocabulaire spécifique requis pour un traducteur-interprète, tout en essayant de montrer et de transmettre l'intérêt et le plaisir que l'on peut en tirer.

Les peuples méditerranéens commencèrent à sortir de la barbarie quand ils apprirent à cultiver l'olivier et la vigne. Justin(2)

Immémoriale, la culture de la vigne est liée à l'activité agricole humaine depuis ses balbutiements. D'abord pratiquée en Europe et en Asie (Moyen-Orient, Caucase), son réel essor est à mettre à l'actif - via les Phéniciens - des Grecs et des Romains qui en développent l'attrait et l'intérêt dans le reste des régions européennes et méditerranéennes quelques siècles avant le début de l'ère chrétienne. En réalité, le vin serait apparu 11000 ans avant notre ère, quand l'oubli supposé de quelques grappes de raisin au fond d'une cruche révélèrent tout l'intérêt de la fermentation de ces fruits, jusque là simplement consommés à maturité. On peut imaginer que les premiers échanges de cette denrée déjà prometteuse ont nécessité le recours à des traducteurs et des interprètes pour transmettre l'ensemble des techniques de culture, de fabrication et de consommation, sans parler de la promotion, de la vente et de l'exportation de ce tout nouveau produit nécessitant déjà des connaissances et un savoir-faire appropriés.

L'art de planter, replanter, tailler et cultiver la vigne fut surtout pratiqué par les Grecs et les Romains qui l'érigèrent en véritable culture dans les deux sens du terme. L'Italie, grâce aux Étrusques, Carthaginois et Romains, fut le premier territoire d'implantation à grande échelle de la vigne et de son commerce. Au fur et à mesure de l'extension de l'Empire romain, des vignobles furent plantés à proximité des lieux de garnison afin d'approvisionner l'armée et de remonter le moral des troupes, marquant incidemment la genèse d'une certaine géographie viticole européenne. Par la suite, l'expansion du christianisme préconisant l'usage du vin autant dans le cadre ecclésiastique que médicinal, contribuera au développement de la viticulture en Europe au cours des premiers siècles de l'ère chrétienne avec l'apparition de nombreux monastères et sites dédiés qui en intensifieront la pratique et la consommation, ainsi que l'influence sociale. Les propriétés aussi multiples que mystérieuses de ce liquide complexe

(2) Historiarum Philippicarum, XLIII. Justin est un historien romain du IIIe siècle. 
justifient d'ailleurs son rôle central dans bien des rites religieux ou païens. L'art comme les représentations historiques et culturelles prendront en outre la vigne comme symbole incontournable de la vie et de la civilisation occidentale.

\section{Présentation botanico-agronomique}

Mais commençons par le début : la vigne (genre botanique vitis) est un arbrisseau ligneux de la famille des Vitaceae (ou Ampélidées) et comporte deux sous-genres déclinés en plusieurs dizaines d'espèces. Elle croît dans les régions chaudes ou tempérées et compte de multiples espèces dont la vitis vinifera, cultivée pour ses fruits en grappes appelés raisins, qui constitue par essence la principale espèce des vignes cultivées en Europe et dans le monde. Le jus de ces raisins possède la propriété naturelle de se transformer en vin après fermentation alcoolique.

Ladite vitis est à l'origine de très nombreux cultivars(3), appelés cépages, qui produisent des vins de différentes qualités dont ils déterminent en grande partie l'arôme et le goût, et dont la complexité demeure encore aujourd'hui mystérieuse, fruit de divers facteurs allant du plant au territoire en passant par le climat, avant même de parler de la vinification.

La vigne préfère les sols caillouteux et poreux qui se réchauffent facilement, tels les terrains siliceux qui produiront des vins plutôt légers, ceux essentiellement argileux qui engendreront des vins colorés, alcooliques, corsés et tanniques, ou encore ceux à dominante calcaire qui donneront naissance à des vins alcooleux(4) et bouquetés(5). En l'occurrence, la célèbre "Côte " bourguignonne réunit les trois, ce qui explique en partie la richesse et la variété de ses vins. La vigne est capable de se développer en maints endroits du monde y compris en des lieux assez inattendus, même s'il convient de reconnaître que les régions où elle est cultivée intensivement se situent entre le 30 e et le 50e parallèle en raison de l'équilibre idéal atteint en termes de température, d'ensoleillement et de précipitations, et du fait que ces contrées ont pour la plupart bénéficié des traditions culturelles et culturales européennes en la matière au cours de leur histoire. Si la vigne est extrêmement sensible au type de sol et au climat, elle possède une surprenante capacité d'adaptation climatique et géographique, au vu des pentes et des hauteurs exceptionnelles qu'elle gravit et atteint dans certaines parties du monde et des fortes amplitudes de température qu'elle supporte pour produire au final d'excellents vins, notamment quand ces conditions naturelles l'obligent à lutter pour survivre. Ainsi, quelques

(3) Type végétal résultant d'une sélection, d'une mutation ou d'une hybridation (naturelle ou provoquée) et cultivé pour ses qualités agricoles.

(4) Alcoolique : qui contient de l'alcool ; alcooleux : qui contient de l'alcool en forte concentration, voire en excès, ce qui est perceptible au nez lors de la dégustation.

(5) Parfumé. 
arpents de vigne judicieusement situés sont susceptibles de créer - avec l'aide de l'homme une cuvée mémorable ! Cette plante constitue donc un spécimen d'étude botanique éminemment intéressant pour la recherche agronomique.

Au fil du temps, la vigne a été introduite sur tous les continents, et la viticulture a pris une réelle importance en Amérique, en Australie, en Afrique du Sud et en Chine, pour ne citer que les régions les plus florissantes de ce qui est communément appelé le "Nouveau Monde ". Elle est aujourd'hui cultivée, sous forme d'alignements, de treilles ou encore de cultures palissées, aux quatre coins de la planète, démontrant ainsi ses qualités d'adaptation géographique, son ancrage historique et son influence culturelle et linguistique. Et pourtant... II s'en est fallu de très peu que son destin, et celui du vin, ne bascule dans l'oubli à la fin du xıxe siècle.

\section{Le phylloxéra, un fait historique déterminant}

Tout le monde ou presque a entendu parler du phylloxéra, responsable de la quasi-éradication des plants de vigne européens. Le Phylloxera vastatrix, improprement nommé à l'origine (parasite des feuilles), est un minuscule mais redoutable insecte découvert peu avant 1870, qui s'attaque aux racines des plants de vigne en se dissimulant dans le sol. Comble d'ironie, il provient du continent américain via des plants importés en Europe à des fins de recherche sur un autre parasite, un champignon appelé oïdium. Ses morsures, mortelles et dévastatrices pour les plants européens (le vignoble français fut réduit de moitié en dix ans), furent heureusement inoffensives pour les plants américains, notamment le typeVitis labrusca. II fut donc procédé, après des dizaines d'années de lutte et de dévastation, à un greffage progressif des plants français sur des porte-greffes américains, résistants, eux, à leur compatriote d'insecte. Autre belle surprise et joli retournement de l'histoire, les plants français greffés ne perdirent aucunement en qualité !

La vigne est une plante qui peut vivre très longtemps. Peu de gens le savent, mais il existe encore quelques rares vignes dites " préphylloxériques ". C'est le cas de la "vigne de la ferme Pédebernade ", située dans le Val d'Adour près du piémont pyrénéen, à Sarragachies (Gers), au cœur de l'appellation Côtes-de-Saint-Mont, et dont la plantation remonte aux environs de l'année 1820. Particularité extraordinaire, elle conserve des pieds non greffés endémiques au piémont pyrénéen ayant échappé au phylloxéra à la fin du xixe siècle, et a même été inscrite au titre des monuments historiques en 2012, une première en France. Par ailleurs, la maison Bollinger, en Champagne, produit une cuvée Vieilles Vignes Françaises à partir d'un vignoble épargné par le phylloxéra. Ces vignes sont franches de pied (non greffées), conduites en foule (à l'alignement aléatoire) et travaillées manuellement. Cette cuvée n'est commercialisée que millésimée, et permet de retrouver le goût du Champagne tel qu'il était au xixe siècle.

Au Portugal, dans la vallée du Douro, une parcelle de vigne appelée La Nacional couvrant 2,5 hectares et produisant le rarissime Porto Vintage Noval Nacional, a elle aussi été épargnée 
par le phylloxéra. En Australie, dans la Barossa Valley, le vignoble de Langmeil planté en 1843 a été classé au Barossa Old Vine Charter pour empêcher son arrachage. Enfin, l'unique pays de culture de vigne au monde où le phylloxéra n'a jamais pénétré est le Chili, où toutes les vignes sont donc "franches de pied " ou " à pied franc ".

Et savez-vous où se trouve la vigne la plus ancienne du monde ? À Maribor en Slovénie, où elle fut plantée il y a... 400 ans. Cette stara trta ("vieille vigne " en slovène) croît le long de la façade de la Maison du vin, musée dédié au vin et à la vigne, et produit 35 à 55 kilos de raisins par vendange dont le vin est conditionné dans une centaine de bouteilles miniatures.

\section{De l'importance du contexte... en viticulture aussi !}

Avant d'entamer la partie purement terminologique de la viticulture, penchons-nous sur les facteurs majeurs influençant les spécificités de la vigne et du vin.

On sait depuis longtemps que le climat influence considérablement le développement de la vigne et donc la qualité gustative du raisin et du vin. Le climat, s'il est essentiel en termes de pluviométrie, de température, d'ensoleillement et de luminosité, ne doit cependant être ni trop froid ni trop chaud, car il influe sur l'acidité et la teneur en sucre du raisin et sur les incontournables polyphénols (molécules organiques présentes dans le règne végétal, et dont le rôle éminent d'antioxydants s'avère extrêmement bénéfique pour la santé), autrefois appelés - et pour cause - tannins végétaux.

Le soleil est un facteur crucial du caractère final du vin. En captant l'énergie solaire, les feuilles de la vigne emmagasinent de la chaleur qui transforme les amidons des feuilles en sucres. Pour schématiser, beaucoup de soleil égale beaucoup de sucre mais insuffisamment d'acidité, sachant que c'est l'acidité qui donne sa structure au vin et que c'est le sucre qui alimente les levures produisant l'alcool. Trop peu de soleil implique un excès d'acidité et un taux insuffisant d'alcool dans le vin.

La pluie, quant à elle, affecte non seulement la quantité mais aussi la qualité du raisin, et en rend les grains gros, fermes et juteux ou petits, resserrés et plus secs selon son abondance. Au moment des vendanges, elle peut se révéler particulièrement calamiteuse en favorisant certaines maladies, champignons ou parasites nocifs, par une humidité permanente sur toutes les parties végétales.

L'emplacement géographique, la composition du sol et la structure géologique constituent les autres facteurs déterminants du développement de la vigne et de la qualité de sa production. En effet, un sol de qualité est la condition sine qua non d'un bon raisin qui est lui-même la condition sine qua non d'un bon vin. Le sol doit être poreux pour favoriser le drainage car si la vigne aime la chaleur, la sécheresse et le soleil, il lui faut un minimum d'eau. D'une façon générale, 
la vigne ne doit pas être prospère, mais plutôt " bonsaïsée(6) " pour l'obliger à chercher dans le sous-sol en profondeur via le système racinaire les divers nutriments indispensables à l'élaboration d'un bon raisin, et donc d'un bon vin.

Enfin, pour terminer cette présentation du cadre naturel, intéressons-nous à cette notion qui prévaut par-dessus tout en viticulture, celle de "terroir ". Ce terme spécifiquement français souvent confondu avec "territoire ", va bien au-delà de la combinaison complexe du sol, du climat, du travail de la vigne (humain ou mécanique) et de l'attention qui lui est prodiguée tout au long de sa croissance. Le mot " terroir " désigne en fait un territoire avec ses particularités physiques, ses productions, ses traditions, sa culture, individuellement et collectivement façonnés par l'activité et le caractère des personnes qui y vivent et y travaillent. Cette notion, essentielle afin d'en rendre tout l'impact et toute la précision dans une traduction selon le contexte, est détaillée dans la partie suivante consacrée à la terminologie.

\section{Balade étymologico-terminologique}

Pour intégrer l'ensemble du vocabulaire de la viticulture, il convient de commencer par quelques remarques d'ordre étymologique. Car si le mot "vigne " désignait à l'origine en latin (vinea) le lieu où l'on cultive la plante (vitis) aujourd'hui éponyme en français, il s'applique désormais autant à la plante qu'à la parcelle ou au domaine où celle-ci est cultivée à grande échelle. Rappelons à ce sujet que le terme "vigne " en français peut revêtir jusqu'à quatre acceptions distinctes : la plante, la parcelle, la souche ou pied et, au pluriel, un domaine planté d'une ou de plusieurs vignes!

Et si les termes dérivés de "vigne " tels que "vignoble " et " vigneron " s'avèrent assez transparents, en revanche, la "vignette ", aux multiples significations qui semblent pourtant bien éloignées du mot "vigne ", en dérive bel et bien. En effet, jusqu'au xvie siècle, on appelait ainsi les ornements en forme de pampres et de ceps dont on décorait les coupes ou les fourreaux d'épée avant que le mot ne passe dans le vocabulaire de l'imprimerie pour désigner de petites gravures ou illustrations et, enfin, dans une évolution plus récente, de petites images séparées, comme les timbres ou les illustrations. Autre méandre étymologique intéressant, la "vis ", d'apparence si commune, vient en droite ligne - si l'on peut dire - de vitis, pour, vous l'aurez compris, la forme vrillée si particulière de son pied appelé cep. Le mot "viniculture ", autrefois synonyme et demeuré quasi-homonyme - trompeur - de "viticulture ", désignait alors l'ensemble des activités ayant pour but la production du vin, englobant la culture de la vigne et la fabrication du vin. Ces termes, apparus dans la terminologie de la vigne et du vin au cours du xıxe siècle, doivent être aujourd'hui dissociés, pour bien distinguer les activités de culture de la vigne (viticulture) et les activités ayant pour but l'élaboration du vin (viniculture). Cette évolution lexicologique a naturellement entraîné l'apparition du terme "vitiviniculture " ou

(6) Taillée comme un bonsaï, arbre maintenu nain en pot par une taille régulière. 
"viti-viniculture " pour désigner la réunion des deux activités que pratique souvent une même personne ou une même entreprise. Les substantifs "viticulteur ", "viniculteur " et les adjectifs " viticole ", " vinicole " et " viti(-)vinicole " procèdent de la même origine lexicologique.

II n'y a de sérieux ici-bas que la culture de la vigne. Voltaire(7)

Pour encore mieux cerner toute la portée de l'activité de la viticulture et son impact crucial sur son produit final, il est pertinent de la décomposer en sept parties distinctes, elles-mêmes composées de termes spécifiques. Ne sont mentionnés ici que les plus spécialisés et les plus évocateurs de la richesse de la terminologie vitivinicole, explicités dans le glossaire qui figure en fin d'article, accompagnés de leurs équivalents anglais et espagnols.

$1 /$ la plantation (défonçage, échalas, plantier, sous-solage),

2/ l'entretien du sol (fumure, millerandage),

3/ le végétal (aoûtement, lignification, bourillon, grume, pruine, sarment, vrille, rafle),

4/ le cycle végétatif (débourrement, effeuillaison, défeuillaison, défoliation, nouaison, véraison, pleurs),

5/ la taille (ébourgeonnage, épamprage, cimage, palissage, rognage, défeuillage, effeuillage, recépage, relevage, éventail, palmette, gobelet),

6/ la multiplication (clone, greffon, porte-greffe, sujet, aoûter),

7/ le traitement des maladies (cochylis, botrytis, coulure, phylloxéra, mildiou, oïdium, pyrale).

Terme ô combien approprié, la " conduite " d'une vigne s'étale sur de nombreuses années. Cruciale parmi les activités précitées, et visant à limiter la croissance naturellement galopante de la vigne, la taille est une opération viticole indispensable qui s'effectue idéalement après les plus fortes gelées, lors du repos végétatif. Elle se décline en une multitude de techniques particulières que recouvrent des termes extrêmement imagés : cordon de Royat, courgées du Jura, gobelet, guyot (simple, double, mixte), lépine, ou encore sylvoz.

Le fruit de la vigne, appelé raisin, peut quant à lui être consommé en tant que fruit frais et se dénomme alors tout

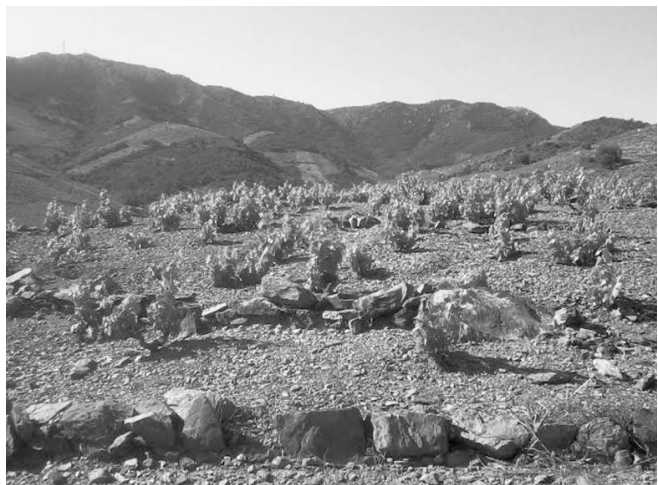

Quand la vigne pousse sur un sol quasi-lunaire...

(Hauteurs de Collioure, P-0).

Photo D. Michelin 24/05/2014

(7) Cité par L. Portes et F. Ruyssen, 1886, Traité de la vigne, 0. Doin, p. 455. 
simplement " raisin de table " ou " raisins secs " si séché, et peut bien sûr être pressé pour fabriquer du jus de raisin. Ce jus instable nécessite toutefois une stérilisation rapide pour être conservé en tant que jus de fruit, voire une transformation pour devenir du vin par un processus de fermentation alcoolique du raisin frais ou du moût de raisin, appelée en ce cas "vinification " ou, s'il est destiné à devenir du vinaigre, " acétification ». Enfin, la " distillation " (procédé permettant d'obtenir de l'alcool en chauffant un liquide faiblement alcoolisé) du vin donne de l'eau-de-vie. Dans ces trois derniers cas, on emploie des cépages spécifiques de "raisin de cuve".

Revenons à la notion de terroir évoquée plus haut, qui n'a pas d'équivalent direct en anglais où le terme français est souvent utilisé pour l'exprimer. L'espagnol, également très riche en terminologie vitivinicole, s'en approche très sensiblement avec les termes terruño, qui signifie à l'origine " pays natal " mais aussi " motte " et traduit très souvent la notion de terroir en viticulture, et pago qui avec un sens original proche s'applique spécifiquement au terrain planté de vignes mais aussi, et c'est éminemment significatif, d'oliviers. Ces deux termes induisent donc une autre nuance en espagnol d'appartenance au sol propre à ces deux cultures.

Pour synthétiser, on peut résumer les effets et propriétés de chaque élément constitutif de la vitiviniculture ainsi :

- Le cépage constitue la garantie des caractéristiques de la variété de raisin.

- Le terroir détermine l'originalité et la spécificité du caractère de la vigne et du vin qu'elle produit.

- Le climat, si optimal et propice à l'extrême, perfectionne l'ensemble pour donner un vin exceptionnel allant jusqu'au grand millésime.

- La vinification procède à la véritable genèse du vin avec tous les facteurs qui y sont liés.

Et si la racine grecque oinos (vin) est réservée aux vocables scientifiques et techniques comme œnologie, œnotourisme, œnophile, œnanthique, œnolisme ou encore œnothèque pour n'en citer qu'une poignée, c'est un vocabulaire majoritairement latin qui forme la base des mots de la vigne et du vin, sans surprise pour une plante et une production d'origine méditerranéenne.

Pour illustrer cette assertion, il s'avère que l'espagnol en tant que langue latine est extraordinairement riche en termes vitivinicoles et surpasse même parfois le français. L'espagnol dispose ainsi d'un terme spécifique pour quatre acceptions différentes désignées en français par le terme unique de "vigne " : vid pour la vigne en tant que plante (pied de vigne), viña pour la vigne en tant que terrain planté de pieds de vignes (parcelle), viñedo pour la vigne en tant que vignoble ou cru, et vidueño pour une vigne plantée d'un cépage unique. Sans parler de la vecera, pied de vigne produisant en alternance d'une année sur l'autre, ou du viñadero, terme ancien désignant la personne qui surveillait les vignes - de jour comme de nuit - dès que le raisin commençait à mûrir afin d'éviter toute intrusion animale ou humaine nuisible à la récolte. Et plus récemment, le terme vineria, surtout usité en Amérique hispanophone et calqué sur le winery américain, qui désigne l'entreprise ou le domaine vitivinicole à grande échelle, pour 
lequel n'existe pas - encore ? - d'équivalent français aussi synthétique. Autre terme spécifique à l'espagnol : canopia, qui désigne l'ensemble des travaux réalisés sur la partie " verte " de la vigne, tels que taille, éclaircissage, rognage et autres activités spécifiques à la viticulture.

Il est intéressant de noter aussi qu'il existe deux termes distincts en espagnol pour la dégustation du vin : cata, où le vin est dégusté en utilisant les sens de façon technique, analytique et objective, et en remplissant une fiche, et degustación, qui n'est pas aussi systématique, moins officiel et laisse place à la perception personnelle. L'espagnol distinguerait donc le plaisir gustatif simple de l'analyse sensorielle technique...

Et sinon, devineriez-vous ce que fait l'" ampélographe " ? Ce terme désigne le métier scientifique qui étudie les caractéristiques des différents types de cépages. Et la tâche semble ardue quand on sait qu'il existe aujourd'hui la bagatelle de 5000 cépages répertoriés dans le monde, qu'ils peuvent être rustiques, productifs, précoces ou tardifs et qu'ils représentent l'équivalent de 40000 dénominations différentes(8) selon les traductions, variantes et adaptations qui leur sont données! Quant au terme " cépage ", il désignait initialement la variété du plant de vigne, et par extension le raisin qu'il produit. II tire son nom du cep, seul et unique terme simple à désigner le pied de vigne, plus particulièrement le tronc, et qui vient du latin cippus, désignant un pieu enfoncé en terre, mais aussi (sic) une colonne funéraire. Enfin, il est intéressant de noter que très peu de langues - a priori-possèdent un mot spécifique unique (non composé ou créé) pour désigner le " cépage ". C'est le cas du français mais aussi de l'italien avec vitigno.

Et quid de " vendange " qui désigne à la fois la récolte du raisin, l'ensemble des raisins récoltés, le millésime, et au pluriel la période de récolte du raisin ? En toute logique, puisque son étymologie latine signifie tout simplement " récolte du vin " (vinum demia puis vindemia). Le " vendémiaire " du calendrier républicain correspond d'ailleurs à cette période de récolte du raisin.

Le " cru » illustre également fort bien la spécificité du vocabulaire français si particulier du domaine, puisqu'il désigne à la fois un vin d'une région ou d'une année en particulier, un terroir ou un vignoble, et ne possède pas non plus d'équivalent direct dans les autres langues.

Et le fameux " chai " (avec ou sans s) ? Si le terme est utilisé à l'origine pour désigner le lieu mythique et souvent historique où sont entreposés et conservés les vins et autres eaux-de-vie dans des tonneaux de différentes sortes, il est aujourd'hui et selon les régions, le lieu destiné à la vinification, à la conservation et/ou l'élevage, et dont l'architecture et la fonction ont considérablement évolué, notamment pour tout ce qui concerne le recyclage de l'eau et des déchets organiques induits. Il est même employé chez certains négociants et exportateurs pour désigner le lieu de départ et de chargement des vins à bord des camions, l'incoterm(9) "ex-works "

(8) La plupart sont des noms propres devenus noms communs ou tirés de termes et toponymes locaux, voire composés (cf. plus bas une sélection de quelques noms originaux).

(9) International commercial term : obligation réciproque du vendeur et de l'acheteur dans le cadre d'un contrat d'achat/vente international. 
(départ entrepôt) devenant en français " ex-chais ». Et à nouveau, l'équivalent exact est quelque peu difficile à trouver en anglais (storehouse) et en espagnol (bodega), car leurs acceptions respectives ne recouvrent pas exactement le même sens.

\section{Exemples concrets de traduction appliquée}

Après cet intermède terminologique, j'aimerais montrer par quelques exemples choisis les difficultés qu'un traducteur peut rencontrer dans la traduction d'un texte de ce domaine et l'importance de connaître et comprendre les termes spécifiques. Les exemples ci-dessous sont empruntés essentiellement à l'univers de la dégustation.

Extraits d'une présentation sur les vins de Pesquera (Cantabrie) au Museo Nacional de Ciencias Naturales (Madrid, 2010):

Tres vinos en el mundo confirman que la grandeza se puede edificar sobre una sola uva: Château Pétrus con merlot, Romanée-Conti con pinot noir y Tinto Pesquera con la tempranillo.

Trois vins au monde prouvent que la grandeur peut se bâtir autour d'un seul cépage : le Château Petrus avec le merlot, le Romanée-Conti avec le pinot noir et le Tinto Pesquera avec le tempranillo.

Noter ici l'emploi du terme " cépage " pour " raisin " (uva) et des noms communs dans les deux langues, et l'utilisation ou non de l'article en espagnol.

Poésie et complexité... Pour les hispanophones et hispanophiles:

Es un vino untuoso, de color caoba oscuro, con profundos aromas de pasificación y paladar aterciopelado.

C'est un vin onctueux, à la teinte acajou sombre, dégageant des arômes intenses de raisins secs et au velouté caractéristique.

Ici, la subtilité porte sur le terme "pasificación " qui désigne la technique du "passerillage " (séchage des raisins sur ou hors pied) et renvoie donc au parfum de raisins secs ou fruits confits.

\section{Extrait d'un communiqué de presse:}

Sourced from some of the best vineyards in the area, this wine is an exceptional new cuvee blend of Chardonnay, Pinot Noir and Pinot Meunier. Particularly sweetened to taste, with some added reserve wines, the new cuvee delivers an intense and full-bodied style.

Issu du cœur des meilleurs vignobles de la région, ce vin incarne une nouvelle cuvée exceptionnelle composée de chardonnay, de pinot noir et de pinot meunier. Harmonieuse combinaison de douceur accentuée par quelques vins de réserve sélectionnés, cette nouvelle cuvée affiche intensité et plénitude. 


\section{Le saviez-vous?}

Le terme chablis, aujourd'hui associé à un vin blanc sec mondialement connu, désignait au départ les arbres, branches et bois renversés par le vent ou affaissés par la neige et le temps, jonchant le sol. D'autres toponymes, plus évidents, ont donné leur nom à des vins : bourgogne, meursault, bordeaux, médoc ou encore chardon(n)ay et chablis, devenus aux États-Unis dans l'usage familier des noms communs désignant de simples vins blancs, forts de cette sonorité française exotique...

Le 25 juillet 1971, l'équipage d'Apollo XV donna officiellement à un cratère lunaire le nom de "Cratère Saint-Georges ", en hommage à Jules Verne, mais aussi au vin de Bourgogne. En effet, dans son roman Autour de la Lune, une fine bouteille de vin de Nuits retrouvée dans le compartiment des provisions est ouverte et dégustée comme il se doit pour fêter " l'union de la Terre et de son satellite ". Le vin a donc depuis longtemps dépassé les frontières planétaires!

La Côte-d'Or est l'unique département français dont le nom ne provient pas d'un critère géographique stricto sensu. Choisi par Charles-André-Rémy Arnoult, avocat au parlement de Dijon et député de l'Assembée constituante de 1789, ce nom s'inspire de la teinte dorée qui recouvre les vignes de la région à l'automne. Le terme " côte " désigne, lui, le relief de la région où poussent ces vignes, constitué par l'affaissement du plateau sédimentaire qui a créé un talus que les géologues désignent sous le terme espagnol de cuesta (" côte " en français). Plus d'un siècle plus tard, ce nom poétique inspira l'écrivain dijonnais Stephen Liégeard qui baptisa une portion du littoral méditerranéen du nom de Côte d'Azur, aujourd'hui définitivement intégré au patrimoine terminologique mondial.

Pour traduire le mot "vigne " (précisément " cep à trois sarments ") dans la Bible en estonien, avant que le vin ne soit importé et la vigne connue dans cette région, un mot dut être créé sur la base de concepts culturels existants. La retranscription littérale donna " arbre à vodka " !

Les fûts gigantesques qui remplissaient autrefois les cales des bateaux s'appelaient les tonnes, d'où dérive le plus petit tonneau. Et si l'usage s'en est perdu de nos jours, leur capacité est demeurée le paramètre mondial de la marine marchande, avec son incontournable " tonnage ".

Le terme claret en anglais (couleur et vin) provient du " clairet " de la région bordelaise, vin rouge traditionnel peu macéré, léger, à fermentation courte et d'une couleur rouge clair, peu chargé en tannins et très aromatique. Énormément apprécié à l'époque de l'Aquitaine anglaise (1152-1453), notamment par la haute société, le claret était exporté vers l'Angleterre par voie maritime. En raison des actes de piraterie sévissant le long des côtes, les bateaux transportant du vin durent être escortés par la marine anglaise et la légende attribue à ces escortes organisées la véritable naissance d'une force militaire marine, à l'origine - se dit-il - de la Royal Navy.

Le célèbre Louis Pasteur fut un grand contributeur de la vinification moderne. Originaire du Jura, seule région de France où les vins sont voilés en surface, à l'instar de ceux de la région de Jerez en Espagne avec leur célèbre "fleur ", il parvint à identifier les micro-organismes qui déclenchent la fermentation du raisin et détermina le rôle de l'oxygène dans la maturation, et son influence sur la couleur du vin, dans son "Étude sur le vin " de 1878. 
Qu'évoquent pour vous Argaman, Aranel, Keknyelu et Kekmedoc? II ne s'agit pas de nouveaux mousquetaires du xxle siècle mais simplement de quelques-uns des plus originaux parmi les innombrables cépages qui existent sur la planète.

Argaman : cépage très foncé, hybride du Carignan et du Sousão portugais, développé à l'origine comme cépage d'assemblage pour teinter les vins rouges trop clairs de cette région. Devenu aujourd'hui un cépage reconnu à part entière. Signifie " pourpre " en hébreu.

Aranel : cépage hybride récent et d'origine obscure, né du croisement de Grenache gris et de Saint-Pierre doré (cépage auvergnat quasi-disparu), aujourd'hui cultivé uniquement dans le sud de la France (Maguelone, Hérault) et en Nouvelle-Galles du Sud en Australie, sur de minuscules parcelles. II serait un parent éloigné du célèbre Chardonnay.

Keknyelu : cépage hongrois, aussi rare qu'insolite, dont les grains mûrs ont la peau verte. Aujourd'hui confiné à l'ouest de la Hongrie, sur la côte nord du lac Balaton, sur une superficie d'à peine 40 hectares, le keknyelu n'est pas hermaphrodite comme la plupart des cépages, et les spécimens mâle et femelle doivent se côtoyer pour se polliniser.

Kekmedoc : cépage hongrois à la peau noire, qui tire son nom du ton bleuté de ses tiges (" kek " signifiant bleu en hongrois) et de l'ajout de "Médoc ", du nom du cépage bordelais [d'origine] dont il provient. Malgré une popularité déclinante, le kekmedoc est un cépage aux nombreuses vertus aromatiques qui produit des vins très rares.

Rkatsiteli : l'un des plus anciens cépages cultivés, originaire de Géorgie, aujourd'hui cultivé en Bulgarie, en Ukraine, en Arménie, en Roumanie et en Moldavie, mais également en Chine (sous le nom de Baiyu) et dans le nord-est des États-Unis où le climat se rapproche de celui de la Géorgie. II se caractérise par sa résistance aux hivers rudes et par sa polyvalence gustative.

Usakhelauri : ancien cépage rare propre à la Géorgie, région viticole la plus ancienne au monde, quasi-exclusif à la région de Racha-Leckhumi, à l'ouest du pays ; il produit une variété de vin demi-doux primé. "Usakhelauri " peut se traduire par " sans nom ", en référence à la qualité exceptionnelle de ses grains qu'aucun mot ne saurait qualifier.

Zlahtina : cépage originaire de la région côtière de Primorska en Croatie, et exclusivement cultivé sur l'île magnifique de Krk (berceau de la langue dalmate aujourd'hui disparue, je laisse les spécialistes en confirmer la prononciation). Caractérisé par de gros grains juteux qui en font un excellent raisin de table et produisent des vins blancs légers, pétillants comme tranquilles, aux arômes d'agrumes, de pomme et de poire. Signifie " noble " en slovène. 


\section{En guise de conclusion...}

Comme on le voit, l'univers de la vigne et du vin - avec toutes ses déclinaisons - couvre un domaine immensément vaste où l'apprentissage - technique comme terminologique - semble infini, et dont l'influence culturelle et linguistique n'est plus à démontrer. Pour preuve, les nombreuses expressions de la langue courante allant du célèbre et intemporel « in vino veritas " des Romains, le français " mettre de l'eau dans son vin ", "quand le vin est tiré, il faut le boire ", "à bon vin, point d'enseigne ", l'espagnol de tal cepa tales sarmiento (tel père, tel fils) ou l'anglais hear it through the grapevine, littéralement " entendre par la vigne ", qui serait une version abrégée de hear it through the grapevine telegraph, par analogie, paraît-il, entre les premières lignes télégraphiques installées de façon rudimentaire sur la côte Est des États-Unis et des pieds de vigne palissés(10)! À l'instar d'autres domaines, la vitiviniculture n'échappe pas à l'essor et au développement récents de technologies avancées appliquées dans la perspective d'une gestion approfondie associant l'amélioration scientifique et technique des pratiques culturales, l'aspect économique (diminution des intrants) et la réduction de l'impact environnemental pour privilégier une qualité de plus en plus sélective et adaptée aux marchés mondiaux. De nouveaux outils technologiques voient le jour et les acteurs du champ terminologique et linguistique doivent aussi s'y adapter, élargissant par là le champ d'action du traducteur.

Par ailleurs, si l'on considère le niveau d'activité et de concurrence inhérent au marché de la vitiviniculture, ses domaines connexes et l'internationalisme qui le caractérise (l'Organisation internationale de la vigne et du vin compte 46 pays membres, nombre en progression constante), la traduction vitivinicole a de beaux jours devant elle et les traducteurs-interprètes de quoi alimenter leur réflexion au gré des multiples vignobles et au fil des innombrables alignements de pieds de vigne de la planète. Sans oublier toute la poésie et la richesse de la langue du vin propre à sa consommation et à sa dégustation car si, selon l'adage, "le bon vin fait parler latin ", il délie aussi bien d'autres langues !

Ainsi, le traducteur spécialisé doit bien sûr répondre à la complexité de certaines traductions, être attentif aux néologismes, gallicismes, régionalismes et autres faux-amis ou synonymes apparents propres au domaine, mais aussi se constituer sa propre base de données à partir des ressources existantes en y intégrant un maximum de détails pratiques et linguistiques. Enfin, la constitution d'un lexique dans ses langues de travail, régulièrement alimenté et actualisé au gré de ses recherches, expériences et rencontres avec des professionnels du secteur à tous les niveaux, est un outil indispensable et constitue le terroir fertile d'une langue spécialisée qui, telle une certaine liane exubérante à la terminologie invasive toujours à domestiquer, ne demande qu'à prospérer.

dommichelin@gmail.com

(10) I heard it through the grapevine est aussi une chanson écrite par Norman Whitfield et Barrett Strong pour Motown Records en 1966, d'abord enregistrée par Smokey Robinson en 1966, puis par Marvin Gaye en 1967, avant d'être commercialisée par Gladys Knight and the Pips, et véritablement popularisée par M. Gaye en 1968 (album In the Groove). 


\begin{tabular}{|c|c|c|c|c|}
\hline Terme & Définition & Anglais & Espagnol & Commentaire \\
\hline Ampélographie & $\begin{array}{l}\text { Science de l'étude de } \\
\text { la vigne et des cépages }\end{array}$ & $\begin{array}{l}\text { Ampelography, } \\
\text { viticultural science }\end{array}$ & Ampelografía & \\
\hline Apyrène (adj.) & $\begin{array}{l}\text { Se dit d'un cépage } \\
\text { produisant des raisins } \\
\text { sans pépins }\end{array}$ & Apirena, seedless & Apirena & \\
\hline Aoûtement & $\begin{array}{l}\text { Processus de lignification } \\
\text { des jeunes rameaux } \\
\text { de la vigne }\end{array}$ & $\begin{array}{l}\text { Lignification, } \\
\text { wood hardening }\end{array}$ & Agostamiento & \\
\hline $\begin{array}{l}\text { Baie } \\
\text { (ou grain de raisin) }\end{array}$ & \begin{tabular}{|l|} 
Fruit de la vigne \\
formant avec la rafle \\
la grappe de raisin, \\
recouvert d'une poussière \\
cireuse protectrice \\
appelée pruine
\end{tabular} & Berry & Baya, grano & $\begin{array}{l}\text { Se compose de } \\
\text { la pellicule, la pulpe } \\
\text { et les pépins }\end{array}$ \\
\hline Botrytis cinerea & $\begin{array}{l}\text { Champignon } \\
\text { microscopique } \\
\text { prospérant sur } \\
\text { la pellicule des raisins }\end{array}$ & Botrytis cinerea & Botrytis cinerea & $\begin{array}{l}\text { Parasite à l'origine } \\
\text { des maladies } \\
\text { communément } \\
\text { appelées } \\
\text { "pourriture " } \\
\text { (noble, grise) }\end{array}$ \\
\hline Cep & Pied de vigne & (vine)stock & Cepa & \\
\hline Cépage & \begin{tabular}{|l|} 
Variété de vigne-- \\
et de raisin - qui se \\
distingue par ses \\
caractéristiques tant \\
botaniques que viniques
\end{tabular} & Variety & Variedad, especie & \\
\hline Chai(s) & $\begin{array}{l}\text { Entrepôt où l'on stockait } \\
\text { les fûts de vin et } \\
\text { d'eau-de-vie en vue } \\
\text { de leur conservation }\end{array}$ & (Wine) storehouse & Bodega & $\begin{array}{l}\text { Par extension, } \\
\text { tout lieu de } \\
\text { conservation et } \\
\text { de stockage du vin }\end{array}$ \\
\hline $\begin{array}{l}\text { Cochylis } \\
\text { ou conchylis }\end{array}$ & $\begin{array}{l}\text { Papillon lépidoptère } \\
\text { dont la chenille dévore } \\
\text { les feuilles de vigne } \\
\text { et perfore les grains } \\
\text { de raisin }\end{array}$ & Cochylis & Cochylis & $\begin{array}{l}\text { Favorise l'installation } \\
\text { de pourritures et } \\
\text { le dessèchement } \\
\text { des grains }\end{array}$ \\
\hline Cordon de Royat & $\begin{array}{l}\text { Type de taille de la vigne } \\
\text { basé sur une seule } \\
\text { charpente à deux bras } \\
\text { en forme de cordon } \\
\text { horizontal }\end{array}$ & $\begin{array}{l}\text { Royat cordon } \\
\text { system of pruning }\end{array}$ & Poda Royat & $\begin{array}{l}\text { Exige une attention } \\
\text { continue }\end{array}$ \\
\hline
\end{tabular}




\begin{tabular}{|c|c|c|c|c|}
\hline Terme & Définition & Anglais & Espagnol & Commentaire \\
\hline Coulure & $\begin{array}{l}\text { Chute accidentelle } \\
\text { (pluie, parasites, maladie) } \\
\text { des fleurs non ou } \\
\text { mal fécondées et/ou } \\
\text { des jeunes fruits lors } \\
\text { de la fécondation }\end{array}$ & Shot berries & $\begin{array}{l}\text { Corrimiento } \\
\text { (caída de la flor) }\end{array}$ & $\begin{array}{l}\text { Affecte le rendement } \\
\text { et l'homogénéité } \\
\text { de la maturation }\end{array}$ \\
\hline Courson & $\begin{array}{l}\text { Portion de bois jeune que } \\
\text { l'on conserve lors de } \\
\text { la taille de la vigne }\end{array}$ & Spur & Pulgar & \\
\hline Cru & $\begin{array}{l}\text { Vin d'une région ou d'une } \\
\text { année en particulier, d'un } \\
\text { terroir ou d'un vignoble }\end{array}$ & Vineyard, vintage & Viñedo, cosecha & \\
\hline $\begin{array}{l}\text { Débourrage/ } \\
\text { débourrement }\end{array}$ & $\begin{array}{l}\text { Période de l'année } \\
\text { où se développent } \\
\text { les bourgeons et où } \\
\text { apparaît la bourre (duvet } \\
\text { et feuilles naissantes) }\end{array}$ & Bud swelling & Desborre, brote & $\begin{array}{l}\text { Marque la fin } \\
\text { de la période dite } \\
\text { de dormance }\end{array}$ \\
\hline $\begin{array}{l}\text { Défeuillaison } \\
\text { (effeuillaison / } \\
\text { effeuillement) }\end{array}$ & $\begin{array}{l}\text { Chute naturelle } \\
\text { des feuilles }\end{array}$ & Leaf fall & $\begin{array}{l}\text { Deshoje, } \\
\text { caída de las hojas }\end{array}$ & \\
\hline $\begin{array}{l}\text { Défoliation / } \\
\text { défeuillage }\end{array}$ & $\begin{array}{l}\text { Destruction des feuilles } \\
\text { au moyen de produits }\end{array}$ & Defoliation & $\begin{array}{l}\text { Defoliación, } \\
\text { deshojado }\end{array}$ & \\
\hline Ébourgeonnage & \begin{tabular}{|l|} 
Suppression \\
des bourgeons superflus
\end{tabular} & Disbudding & Desyemado & \\
\hline Échalas & $\begin{array}{l}\text { Pieu servant de support } \\
\text { au cep de vigne }\end{array}$ & Stick, stake, cane & Estaca, rodrigón & \\
\hline Effeuillage & $\begin{array}{l}\text { Suppression des vieilles } \\
\text { feuilles pour favoriser } \\
\text { l'ensoleillement } \\
\text { des grappes }\end{array}$ & Leaf removal & $\begin{array}{l}\text { Manejo de follaje, } \\
\text { aclarado, clareo }\end{array}$ & \\
\hline Épamprage & $\begin{array}{l}\text { Suppression des pampres } \\
\text { (rameaux non fructifères), } \\
\text { bourgeons et feuilles } \\
\text { inutiles (gourmands) }\end{array}$ & Suckering & $\begin{array}{l}\text { Despampano, } \\
\text { despampanado }\end{array}$ & \\
\hline Éventail (taille en) & $\begin{array}{l}\text { Taille caractérisée par } \\
\text { un cep court et des bras } \\
\text { longs s'écartant vers le } \\
\text { haut dans toutes les } \\
\text { directions }\end{array}$ & $\begin{array}{l}\text { Fan-shaped } \\
\text { (pruning) }\end{array}$ & Poda en abanico & $\begin{array}{l}\text { Implique une récolte } \\
\text { manuelle }\end{array}$ \\
\hline
\end{tabular}




\begin{tabular}{|c|c|c|c|c|}
\hline Terme & Définition & Anglais & Espagnol & Commentaire \\
\hline Fleur & \begin{tabular}{|l|} 
Voile blanchâtre, plus \\
ou moins ridé, \\
se formant en cuve ou \\
en bouteille à la surface \\
des vins au contact de \\
l'air, généré par \\
les levures
\end{tabular} & Coating & Flor & \\
\hline $\begin{array}{l}\text { Franc de pied } \\
\text { (à pied franc) }\end{array}$ & Cep de vigne non greffé & Ungrafted & $\begin{array}{l}\text { Franco de pie / } \\
\text { en pie franco }\end{array}$ & $\begin{array}{l}\text { La tendance est au } \\
\text { retour progressif de } \\
\text { ces plants dans } \\
\text { certaines régions }\end{array}$ \\
\hline Gobelet (taille) & $\begin{array}{l}\text { Taille caractérisée par } \\
\text { un cep court et des bras } \\
\text { longs disposés sur un } \\
\text { plan vertical ou oblique }\end{array}$ & $\begin{array}{l}\text { Open-centred } \\
\text { (pruning) }\end{array}$ & $\begin{array}{l}\text { Poda en vaso } \\
\text { (o copa) }\end{array}$ & $\begin{array}{l}\text { Taille simple et } \\
\text { permettant une } \\
\text { aération optimale }\end{array}$ \\
\hline Greffon & $\begin{array}{l}\text { Branche ou bourgeon } \\
\text { implanté sur une autre } \\
\text { plante (porte-greffe) } \\
\text { pour réaliser une greffe }\end{array}$ & Scion, shoot, graft & Injerto & \\
\hline Lignification & $\begin{array}{l}\text { Transformation } \\
\text { du végétal en bois }\end{array}$ & Lignification & Lignificación & $\begin{array}{l}\text { Le tissu végétal } \\
\text { s'imprègne de lignine, } \\
\text { constituant essentiel } \\
\text { du bois }\end{array}$ \\
\hline Mildiou & $\begin{array}{l}\text { Maladie fongique } \\
\text { affectant notamment } \\
\text { les feuilles de la vigne } \\
\text { qui se recouvrent } \\
\text { d'un duvet blanchâtre }\end{array}$ & Mildew & Mildiú & \\
\hline Millerandage & $\begin{array}{l}\text { Fécondation imparfaite } \\
\text { des fleurs qui donneront } \\
\text { des baies sans pépin } \\
\text { (ou apyrènes) qui } \\
\text { restent petites, } \\
\text { sont plus sucrées } \\
\text { et moins acides }\end{array}$ & Millerandage & $\begin{array}{l}\text { Millerandage, } \\
\text { corrimiento }\end{array}$ & \\
\hline Millésime & $\begin{array}{l}\text { Année de la récolte } \\
\text { du raisin ayant servi } \\
\text { à produire un vin }\end{array}$ & Vintage & Añada & \\
\hline
\end{tabular}




\begin{tabular}{|c|c|c|c|c|}
\hline Terme & Définition & Anglais & Espagnol & Commentaire \\
\hline Moût & $\begin{array}{l}\text { Jus de raisin non } \\
\text { fermenté composé de } \\
\text { jus, de pulpe, de peau, } \\
\text { de pépins et de rafles }\end{array}$ & Must & Mosto & $\begin{array}{l}\text { Constitue la matière } \\
\text { première de } \\
\text { la vinification }\end{array}$ \\
\hline Nouaison / nouure & $\begin{array}{l}\text { Phase initiale de la } \\
\text { formation du fruit après } \\
\text { fécondation de la fleur }\end{array}$ & Fruit set(ting) & $\begin{array}{l}\text { Cuajado, } \\
\text { fructificación }\end{array}$ & \\
\hline OEnanthique & Relatif à l'arôme des vins & Oenanthic/enanthic & Enantico & \\
\hline Oidium & Maladie causée à la vigne & Powdery mildew & $\begin{array}{l}\text { Oídio, } \\
\text { mildiú polvoroso }\end{array}$ & \\
\hline Palissage & $\begin{array}{l}\text { Maintien de la végétation } \\
\text { de la vigne sur un plan } \\
\text { vertical à l'aide } \\
\text { de tuteurs et/ou de fils }\end{array}$ & $\begin{array}{l}\text { Trellising, vine tying, } \\
\text { training }\end{array}$ & Empalizamiento & \\
\hline Pampre & $\begin{array}{l}\text { Rameau ou jeune } \\
\text { pousse de vigne } \\
\text { de l'année (non lignifiée) }\end{array}$ & (young) vine branch & Pámpano & $\begin{array}{l}\text { Par extension, } \\
\text { ornement en forme } \\
\text { de rameau de vigne }\end{array}$ \\
\hline Passerillage & $\begin{array}{l}\text { Technique vinicole qui } \\
\text { s'utilise dans certaines } \\
\text { régions viticoles, pour } \\
\text { enrichir le raisin en sucre } \\
\text { par concentration, par } \\
\text { ensoleillement et/ou } \\
\text { séchage des grappes } \\
\text { sur ou hors souche }\end{array}$ & $\begin{array}{l}\text { Raisining, } \\
\text { grape-wilting, } \\
\text { withering, } \\
\text { dessication }\end{array}$ & Pasificación & $\begin{array}{l}\text { Cette technique } \\
\text { (hors souche) } \\
\text { permet d'élaborer } \\
\text { notamment les vins } \\
\text { dits " de paille " }\end{array}$ \\
\hline Plantier & Nouveau plant de vigne & New vine(yard) & Nueva viña & $\begin{array}{l}\text { Par extension, terrain } \\
\text { nouvellement planté } \\
\text { de vigne }\end{array}$ \\
\hline Pleurs & $\begin{array}{l}\text { Écoulements de sève } \\
\text { sur des coupes non } \\
\text { cicatrisées dus } \\
\text { à une taille tardive }\end{array}$ & Bleeding & Llantos & \\
\hline Porte-greffe & $\begin{array}{l}\text { Plant de vigne } \\
\text { supportant le greffon }\end{array}$ & Rootstock & Portainjerto & \\
\hline Pressoir & $\begin{array}{l}\text { Dispositif servant } \\
\text { à presser le raisin }\end{array}$ & $\begin{array}{l}\text { Winepress / } \\
\text { Pressroom }\end{array}$ & $\begin{array}{l}\text { Lagar (double } \\
\text { sens également) }\end{array}$ & $\begin{array}{l}\text { Par extension, } \\
\text { pièce où se trouve } \\
\text { ledit pressoir }\end{array}$ \\
\hline
\end{tabular}




\begin{tabular}{|c|c|c|c|c|}
\hline Terme & Définition & Anglais & Espagnol & Commentaire \\
\hline Pruine & $\begin{array}{l}\text { Couche cireuse présente } \\
\text { sur la pellicule du raisin }\end{array}$ & Bloom & Pruina & $\begin{array}{l}\text { Intègre les levures } \\
\text { et moisissures } \\
\text { nécessaires à } \\
\text { la fermentation } \\
\text { et sert également } \\
\text { de protection } \\
\text { aux agressions } \\
\text { bactériennes }\end{array}$ \\
\hline Pyrale & $\begin{array}{l}\text { Insecte lépidoptère } \\
\text { de type papillon } \\
\text { dont la chenille } \\
\text { s'attaque à la vigne }\end{array}$ & Codling moth & Piral & \\
\hline $\operatorname{Raf}(f) l e$ & $\begin{array}{l}\text { Ensemble } \\
\text { du pédoncule (queue) } \\
\text { et des pédicelles } \\
\text { (ramifications) } \\
\text { soutenant les grains } \\
\text { du raisin }\end{array}$ & Stalk & Raspón & \\
\hline Recépage & $\begin{array}{l}\text { Action de couper } \\
\text { un végétal près du sol } \\
\text { pour favoriser } \\
\text { la (re)pousse }\end{array}$ & $\begin{array}{l}\text { Cutting back, } \\
\text { coppicing, } \\
\text { bottom pruning }\end{array}$ & $\begin{array}{l}\text { Tala a ras de tierra, } \\
\text { rebrote }\end{array}$ & \\
\hline Relevage & $\begin{array}{l}\text { Opération consistant } \\
\text { à relever les sarments } \\
\text { pour les guider } \\
\text { à l'intérieur des fils } \\
\text { de palissage }\end{array}$ & Lifting & Elevación & \\
\hline Rognage & $\begin{array}{l}\text { Réduction de l'extrémité } \\
\text { des rameaux de vigne } \\
\text { visant à équilibrer } \\
\text { la végétation } \\
\text { et le développement } \\
\text { du raisin }\end{array}$ & Cutting & $\begin{array}{l}\text { Recorte, } \\
\text { cercenadura }\end{array}$ & \\
\hline Sarment & $\begin{array}{l}\text { Branche de vigne } \\
\text { de l'année lignifiée } \\
\text { que l'on coupe } \\
\text { après les vendanges }\end{array}$ & Cane & Sarmiento & \\
\hline
\end{tabular}




\begin{tabular}{|l|l|l|l|l|}
\hline \multicolumn{1}{|c|}{ Terme } & \multicolumn{1}{|c|}{ Définition } & \multicolumn{1}{c|}{ Anglais } & \multicolumn{1}{c|}{ Espagnol } & \multicolumn{1}{c|}{ Commentaire } \\
\hline Souche & $\begin{array}{l}\text { Partie du pied de vigne } \\
\text { situé entre le greffon } \\
\text { et les racines } \\
\text { du porte-greffe }\end{array}$ & Stock & Cepa & $\begin{array}{l}\text { C'est à partir de la } \\
\text { souche qu'est } \\
\text { comptabilisé le } \\
\text { rendement à la } \\
\text { parcelle (nombre de } \\
\text { souches x nombre de } \\
\text { grappes x poids } \\
\text { moyen d'une grappe } \\
\text { divisé par la surface } \\
\text { de la parcelle) }\end{array}$ \\
\hline Tan(n)in & $\begin{array}{l}\text { Substance végétale } \\
\text { contenue dans la peau, } \\
\text { le pépin } \\
\text { et la rafle du raisin }\end{array}$ & Tannin & Tanino & \\
\hline Sous-solage & $\begin{array}{l}\text { Ameublissement profond } \\
\text { du sol sans } \\
\text { retournement de la terre }\end{array}$ & Subsoiling & Mullimiento & \\
\hline Véraison & $\begin{array}{l}\text { Couleur que prennent } \\
\text { les raisins lorsqu'ils } \\
\text { arrivent à maturité, } \\
\text { et par extension période } \\
\text { correspondante }\end{array}$ & $\begin{array}{l}\text { (grape) coloring, } \\
\text { ripening }\end{array}$ & Envero & $\begin{array}{l}\text { Erte } \\
\text { et des plantes } \\
\text { grimpantes qui permet } \\
\text { de s'agripper } \\
\text { à un support }\end{array}$ \\
\hline Vrille & Tendril & Zarcillo & $\begin{array}{l}\text { Provient de la même } \\
\text { racine que « vigne " } \\
\text { (vitis) }\end{array}$ \\
\hline
\end{tabular}

\section{Bibliographie et références}

JOHNSON Hugh, 2002, Une histoire mondiale du vin, de l'Antiquité à nos jours, Paris, Hachette. ARGOD-DUTARD Françoise, CHARVET Pascal et LAVAUD Sandrine (dir.), 2007, Voyage aux pays du vin, Paris, R. Laffont, Bouquins.

COUTIER Martine, 2013, Le dictionnaire de la langue du vin, Paris, CNRS, Biblis.

COLLOMBET François, 2014, Les vignobles mythiques, Paris, Belin.

VANDYKE PRICE Pamela, 1986, Le vrai livre du vin, Tours (France), Princesse.

BELLOS David, 2013, Le poisson et le bananier, une histoire fabuleuse de la traduction, Paris, Flammarion.

GALET Pierre, 2000, Dictionnaire encyclopédique des cépages, Paris, Hachette.

MAC NEIL Karen, 2001, The Wine Bible, New York (États-Unis), Workman. 


\section{Sitographie}

http://www.cepdivin.org/actu/parutions/voyage_aux_pays_du_vin.html (consulté le 8/8/14). http://www.oiv.int/oiv/info/fr (consulté le 10/8/14).

http://www.interfrance.com/fr/vin/wine-terms.html (consulté à diverses reprises entre août et septembre 2014).

http://www.dico-du-vin.com/ (consulté à diverses reprises entre août et septembre 2014).

http://www.linternaute.com/voyage/magazine/dossier/sur-la-route-des-vins-du-monde/un-tourdu-monde-des-vins.shtml (consulté le 28/09/14).

Dominic Michelin, traducteur indépendant depuis quatre ans, est installé en Côte-d'Or. De formation littéraire et linguistique, il a précédemment travaillé dans le commerce international et la communication multiculturelle. II a notamment été responsable de la communication et traducteur dans le domaine du transport aérien et de l'aéronautique, de l'action humanitaire et en agronomie, et enseignant en français langue étrangère (Canada, Australie). Diplômé de l'université de Dijon-Bourgogne (Master en Stratégies de communication internationale, 2001), de l'université Paris VII et de I'ISIT (Master en Industrie des langues et Traduction spécialisée, 2010). Lauréat du concours de traduction "On traduit dans l'Estrie " 2012. 